\title{
Unexplained anemia in the elderly - a real life analysis of 981 patients
}

Sylwia S. Michalak ${ }^{1}$, Joanna Rupa-Matysek², Iwona Hus ${ }^{3}$, Lidia Gil²

${ }^{1}$ Department of Pharmacology and Toxicology, Faculty of Medicine and Health Science, University of Zielona Gora, Zielona Gora, Poland 2Department of Hematology and Bone Marrow Transplantation, Poznan University of Medical Sciences, Poznan, Poland

${ }^{3}$ Department of Clinical Transplantology, Medical University of Lublin, Lublin, Poland

Submitted: 2 December 2018

Accepted: 6 January 2019

Arch Med Sci 2020; 16 (4): 834-841

DOI: https://doi.org/10.5114/aoms.2019.82723

Copyright $\odot 2019$ Termedia \& Banach

\section{Abstract}

Introduction: We aimed to analyze the prevalence of unexplained anemia (UA) and assess its characteristics, potential causes and impact on survival in an elderly population.

Material and methods: Medical files of 981 patients aged $\geq 60$ years consulted in one primary medical clinic in Poland in 2013-2014 were retrospectively analyzed. Anemia, defined according to WHO criteria, diagnosed during either hospitalization or outpatient treatment, from the age of 60 , was included. Unexplained anemia was diagnosed if, based on available clinical data and laboratory tests and other assessments in medical records, none of the well-known types of anemia were identified.

Results: Of 981 patients with anemia, UA was found in 48 (28.4\%) patients ( $4.9 \%$ of those studied) and incidence increased with age ( $\geq 80$ years, $12.3 \%$ ). In $81.3 \%$ no full hematological diagnostics were performed. Patients with UA, as with those with defined anemia, when compared to the group without anemia were older, had more co-morbidities, were more frequently hospitalized, more frequently had dementia syndrome and obtained lower Barthel scores $(p<0.0001)$. In the groups of patients with UA and defined anemia, there were more deaths than in those without anemia (10\% vs. $13 \%$ vs. $2 \%, p<0.0001)$ with significant differences in survival rates observed during 3-year follow-up.

Conclusions: The increasing incidence with age of UA in the elderly population, insufficient diagnosis and the higher mortality of patients with UA in comparison to the group without anemia indicate the need to develop recommendations for its management by primary care physicians.

Key words: elderly, survival, unexplained anemia.

\section{Introduction}

Anemia is a significant problem in the elderly population with a proven negative impact on the course of co-morbidities and survival [1-3]. The estimated prevalence of anemia in the population over 60 years old ranges from $2.9 \%$ to $61 \%$ in men and from $3.3 \%$ to $41 \%$ in women [4]. In $16-40 \%$ of cases, the etiology of anemia remains unexplained (UA), despite thorough analysis of the medical history, comorbidities, physical examination and appropriate laboratory tests, including bone marrow evaluation [5-10]. Currently, based on extended molecular studies it is

\author{
Corresponding author: \\ Sylwia S. Michalak PhD \\ Department \\ of Pharmacology \\ and Toxicology \\ Faculty of Medicine \\ and Health Science \\ University of Zielona Gora \\ 28 Zyty St \\ 65-046 Zielona Gora, Poland \\ Phone: +48 502857453 \\ E-mail: s.michalak@wlnz. \\ uz.zgora.pl
}


suggested that this group of patients be designated as having cytopenia of unknown significance with isolated anemia (ICUS-A) [11, 12]. However, the scope and qualification of elderly patients for specific diagnostic procedures are still being discussed in the literature $[11,13,14]$. Additionally, there are conflicting reports on the impact of UA on the survival of patients $[8,13,14]$. There are no studies assessing the scope of diagnosis and characteristics of patients with UA as a real-life analysis.

Therefore, the aim of this study was to assess the prevalence of unexplained anemia in the Polish population aged $\geq 60$ years, its characteristics, an assessment of potential causes (along with the scope of the performed diagnostics) and the impact of UA on patients' survival.

\section{Material and methods}

We retrospectively analyzed the medical files of 981 patients aged $\geq 60$ years consulted in one primary medical care clinic in Poland in 2013-2014 for the occurrence of anemia, comorbidities and hospitalizations. All available laboratory tests of differential diagnosis of anemia (including bone marrow examination, cytogenetic/molecular tests) and medical records concerning comorbidities, hospitalizations since the age 60 and deaths were evaluated [15].

Anemia was diagnosed according to the criteria of the World Health Organization for individuals aged $\geq 65$ years $(\mathrm{Hb}<12 \mathrm{~g} / \mathrm{dl}$ in women, $\mathrm{Hb}<13 \mathrm{~g} / \mathrm{dl}$ in men) [16]. Four degrees of severity were determined: mild anemia (10-12 g/dl in women, $10-13 \mathrm{~g} / \mathrm{dl}$ in men), moderate $(8-9.9 \mathrm{~g} / \mathrm{dl})$, severe $(6.5-7.9 \mathrm{~g} / \mathrm{dl})$ and very severe anemia $(<6.5 \mathrm{~g} / \mathrm{dl})$. Macrocytosis was diagnosed as mean cell volume (MCV - the mean volume of a red blood cell) $>100 \mathrm{fl}$, normocytosis as MCV 80-100 fl and microcytosis as MCV < $80 \mathrm{fl}$. Leukopenia was defined as white blood cell (WBC) count $<4 \times 10^{9} /$, thrombocytopenia as platelet (PLT) count $<140 \times 10^{9} / \mathrm{l}$.

Patients with anemia were divided into two groups: with defined anemia (DA) and with UA. The group of defined anemia included: deficiency anemia (iron, vitamin $B_{12}$, folic acid deficiency), bleeding anemia, anemia of chronic disease, hemolytic anemia, aplastic anemia, chronic kidney disease anemia (CKD), chemo- and/or radiotherapy induced anemia, anemia in the course of chronic liver disease, alcoholic disease and thyroid disease. We used the definitions for the particular types of anemia described previously [15]. Anemia was defined as UA if, based on the available clinical data, laboratory tests and other assessments/ records in the medical files, none of the abovementioned types of anemia were identified. Diagnosis of anemia was determined as incomplete when the obtained laboratory tests were unable to exclude all defined types of anemia [15]. In the group of patients with UA, the duration of anemia from its diagnosis to the end of observation was evaluated. Furthermore, the presence of anemia before the age of 60 in this group was also studied. The frequency of the Barthel test performance and the scores of the Barthel scale were examined as part of the geriatric evaluation [17]. The Bioethical Committee of Poznan University of Medical Sciences approved the study in accordance with the Declaration of Helsinki.

\section{Statistical analysis}

The descriptive statistics of the study group, such as gender and deaths, were expressed as percentages and numbers. The Shapiro-Wilk test was performed to assess normality, whereas the homogeneity of variance was evaluated by Levene's test. Variables that had a normal distribution and low validity were represented by the mean and standard deviation. Otherwise, the median and standard error were used.

Comparative analysis between the analyzed groups of anemia was performed for gender, deaths, comorbidities and hospitalization using the Pearson $\chi^{2}$ test with analyses of structural indices between the analyzed groups, whereas statistically significant differences of age, laboratory parameters and Barthel scale between the analyzed groups of anemia were examined using Kruskal-Wallis one-way analysis of variance by ranks together with the post hoc Z test for multiple comparisons.

To evaluate differences in the laboratory data of the analyzed groups with anemia or without anemia, effect size was calculated as the difference between means divided by the pooled standard deviation. Using Cohen's D criteria, an effect size $>0.20$ and $<0.50$ was considered small, $\geq 0.50$ and $<0.80$ medium, and $\geq 0.80$ large. The probabilities of survival were estimated via the Kaplan-Meier method, and comparisons were performed via the $\chi^{2}$ test. A $p$-value below 0.05 was regarded as statistically significant. The statistical analyses were performed with Statistica 13 (StatSoft, Inc. 2017 software, Tulsa, Oklahoma, USA).

\section{Results}

In the analyzed population of 981 individuals at the age of $\geq 60$ years or older, anemia was diagnosed in 169 (17.2\%) patients. UA was found in 48 patients ( 27 women and 21 men), which accounted for $4.9 \%$ of the entire study population and $28.4 \%$ of those with anemia. Analysis according to the age groups demonstrated that UA occurred in $2.6 \%$ of people aged $60-69$, in $5.1 \%$ aged $70-79$ and in $12.3 \%$ in the $\geq 80$ years age group. The frequency was higher in the group aged 70-79 vs. 60-69 years (ns) and significantly higher in the 
Table I. Characteristics of patients with UA, DA or without anemia

\begin{tabular}{|c|c|c|c|c|c|}
\hline Characteristics & $\begin{array}{l}\text { Whole } \\
\text { population } \\
(n=981)\end{array}$ & $\begin{array}{l}\text { Unex- } \\
\text { plained } \\
\text { anemia }^{\mathrm{e}} \\
(n=48)\end{array}$ & $\begin{array}{l}\text { Defined } \\
\text { anemia }^{c, d} \\
(n=121)\end{array}$ & $\begin{array}{c}\text { No anemia } \\
\text { group }{ }^{\mathrm{a}, \mathrm{b}} \\
(n=812)\end{array}$ & $P$-value \\
\hline Female, $n(\%)$ & $594(61)$ & $27(56)$ & $52(63)$ & $504(62)$ & 0.09 \\
\hline Age, mean \pm SD [years] & $70.8 \pm 8.45$ & $75.9 \pm 9.2$ & $74.7 \pm 9.3$ & $69.8 \pm 8.0$ & $<0.0001$ \\
\hline $\mathrm{Hb}$, mean $\pm \mathrm{SD}[\mathrm{g} / \mathrm{dl}]$ & $13.47 \pm 1.79$ & $11.2 \pm 1.2$ & $10.2 \pm 1.6$ & $14.09 \pm 1.06$ & $<0.0001$ \\
\hline $\mathrm{WBC}$, mean $\pm \mathrm{SD}\left[\times 10^{9} / \mathrm{I}\right]$ & $6.94 \pm 2.77$ & $7.08 \pm 3.29$ & $7.93 \pm 5.74$ & $6.78 \pm 1.90$ & 0.63 \\
\hline $\mathrm{PLT}$, mean $\pm \mathrm{SD}\left[\times 10^{9} / \mathrm{I}\right]$ & $234.6 \pm 71.6$ & $231.7 \pm 82.3$ & $269.9 \pm 117.9$ & $229.5 \pm 59.4$ & 0.01 \\
\hline eGFR, , mean $\pm \mathrm{SD}\left[\mathrm{ml} / \mathrm{min} / 1.73 \mathrm{~m}^{2}\right]$ & $81.4 \pm 22.9$ & $74.4 \pm 26.5$ & $70.7 \pm 28.1$ & $83.4 \pm 21.3$ & $<0.0001$ \\
\hline $\mathrm{CRP}$, mean $\pm \mathrm{SD}[\mathrm{mg} / \mathrm{l}]$ & $24.3 \pm 51.8$ & $25.7 \pm 80.6$ & $42.9 \pm 60.0$ & $6.2 \pm 14.6$ & $<0.0001$ \\
\hline Number of comorbidities median (range) & $2(0-12)$ & $4(1-7)$ & $4(0-9)$ & $2(0-8)$ & $<0.0001$ \\
\hline Number of hospitalizations median (range) & $0(0-12)$ & $1(0-8)$ & $2(0-12)$ & $0(0-11)$ & $<0.0001$ \\
\hline Barthel scale, mean \pm SD & $85 \pm 12.3$ & $80 \pm 14.9$ & $80 \pm 16.2$ & $85 \pm 8.4$ & $<0.0001$ \\
\hline Death, $n(\%)$ & $40(4)$ & $5(10)$ & $16(13)$ & $19(2)$ & $<0.0001$ \\
\hline
\end{tabular}

${ }^{a}$ A significantly greater value in the NA group than the UA group and the NA group than the DA group for the following parameters: Hb, eGFR and Barthel scale scores. ${ }^{b} A$ significantly lower value in the NA group than the UA group and the NA group than the DA group for the following parameters: number of hospitalizations, number of comorbidities in patients. 'A significantly greater value in the DA group than the NA group for the following parameters: PLT, CRP, age and deaths. ${ }^{~} A$ significantly greater value in the DA group than the UA group for the following parameters: CRP and PLT. ${ }^{e} A$ significantly greater value in the UA group than the NA group for the following parameters: age and deaths. $C R P-C$ reactive protein, DA - defined anemia, eGFR - estimated glomerular filtration rate, Hb-hemoglobin, NA-no anemia, PLT-platelet count, UA - unexplained anemia, WBC - white blood cell count. A p-value less than 0.05 was considered statistically significant.

group aged $\geq 80$ vs. $60-69$ years $(p<0.0001)$ and the group aged $\geq 80$ vs. $70-79$ years $(p=0.007)$.

Patients with UA were older than those without anemia $(75.9 \pm 9.2$ vs. $69.8 \pm 8.0$ years, $p<0.0001)$. In the group of patients with UA, as well as in the group with DA, there were significantly more deaths than in the group without anemia: $10 \%$ vs. $13 \%$ vs. $2 \%(p<0.0001)$, respectively (Table I).

\section{Characteristics of patients with anemia}

In most of the patients with UA the severity of anemia was mild $(\mathrm{Hb} \geq 10 \mathrm{~g} / \mathrm{dl})$, as shown in Figure 1 . There were no cases of very severe anemia in the UA group.

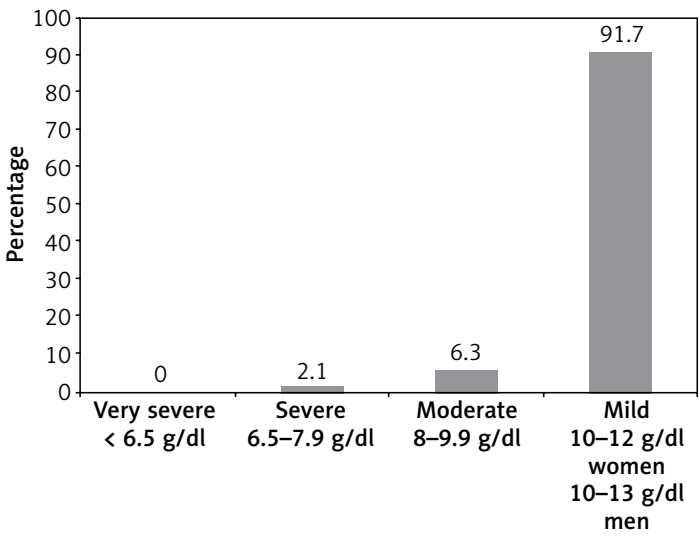

Figure 1 . Severity of anemia in patients with unexplained anemia
The mean $\mathrm{Hb}$ concentration in women with UA was $10.7 \pm 1.2 \mathrm{~g} / \mathrm{dl}$, while in men it was 11.9 $\pm 0.8 \mathrm{~g} / \mathrm{dl}$. Mild anemia was found in $100 \%$ of men and $85 \%$ of women $(p=0.06)$. Analysis of MCV in the UA group showed that $70.8 \%$ of patients had normocytosis and $4.2 \%$ had macrocytosis. In $11(22.9 \%)$ patients with UA, there were no data on MCV.

In 39 (81.3\%) patients in the UA group, detailed hematologic diagnostics were not performed, including bone marrow or cytogenetic/molecular tests. The lack of diagnostics was significantly more frequent in the group aged $60-69$ vs. 70-79 years and $60-69$ vs. $\geq 80$ years ( $p=0.03$ and $p=$ 0.01 , respectively). In $8(16.7 \%)$ patients surgery was performed within 3 months before the diagnosis of anemia. It was performed more often in patients aged $60-69$ vs. $\geq 80$ years $(p=0.01)$. In 9 (18.8\%) patients, anemia was accompanied by other hematological abnormalities, including $7(14.6 \%)$ patients with a WBC count below $4 \times$ $10^{9} / \mathrm{l}$ or a PLT count below $140 \times 10^{9} / \mathrm{l}$, while in 2 (4.2\%) macrocytosis (MCV > $100 \mathrm{fl}$ ) was found. No patients with UA had pancytopenia with concurrent macrocytosis, whereas 2 patients with UA had a reduced PLT count below $140 \times 10^{\%} / /$ and WBC below $4 \times 10^{9} / \mathrm{l}$. Macrocytosis was significantly more common in the patients aged 60-69 years as compared to the groups aged $70-79$ years $(p=0.004)$ and $60-69$ vs. $\geq 80$ years $(p=0.003)$. 
The mean age of diagnosis of UA was $73.9 \pm 10.1$ years. Only in 3 patients did UA occur before the age of 60 . The average duration of UA from diagnosis to the end of follow-up was $36 \pm 48$ months, median: 19 months (range: 2-271 months).

There were statistically significant differences between the analyzed groups for the following parameters: hemoglobin, platelet count, estimating glomerular filtration rate (eGFR), C-reactive protein (CRP), age, number of comorbidities, number of hospitalizations, Barthel scale score and deaths (Table I). Patients with UA had lower
eGFR when compared to the group without anemia, while higher CRP was found in the group with DA as compared to the patients with UA. There were significant differences in the clinical characteristics between patients without anemia and patients with UA or DA. Patients with UA when compared to the group without anemia had more comorbidities, were more frequently hospitalized and presented lower scores on the Barthel scale (Table I). The Barthel scale was performed on 137 (81\%) patients with anemia, and in 265 (32.6\%) cases without anemia.

Table II. Analysis of co-morbidities and the use of selected groups of drugs and performed procedures in patients with unexplained anemia, defined anemia and no anemia

\begin{tabular}{|c|c|c|c|c|c|}
\hline Characteristics & $\begin{array}{c}\text { Whole } \\
\text { population } \\
(n=981)\end{array}$ & $\begin{array}{l}\text { Unex- } \\
\text { plained } \\
\text { anemia }{ }^{\mathrm{a}, \mathrm{b}} \\
(n=48)\end{array}$ & $\begin{array}{l}\text { Defined } \\
\text { anemia }^{d} \\
(n=121)\end{array}$ & $\begin{array}{c}\text { No anemia } \\
\text { group }^{c} \\
(n=812)\end{array}$ & $P$-value \\
\hline \multicolumn{6}{|l|}{ Comorbidities, \% (n): } \\
\hline Hypertension & 78.7 (772) & $95.8(46)$ & $82.6(100)$ & $77.1(626)$ & 0.005 \\
\hline Coronary heart disease & $32.5(319)$ & $62.5(30)$ & $38.0(46)$ & $29.9(243)$ & $<0.0001$ \\
\hline Diabetes & $25(245)$ & $35.4(17)$ & $29.7(36)$ & $23.6(192)$ & 0.08 \\
\hline Chronic kidney disease & $12.1(102)$ & $29.6(13)$ & $34.6(36)$ & $7.6(53)$ & $<0.0001$ \\
\hline Heart failure & $10.8(106)$ & $31.2(15)$ & $23.1(28)$ & $7.8(63)$ & $<0.0001$ \\
\hline Atrial fibrillation & $10.4(102)$ & $20.8(10)$ & $20.7(25)$ & $8.2(67)$ & $<0.0001$ \\
\hline Rheumatic diseases & $1.1(11)$ & $2.1(1)$ & $5.0(6)$ & $0.5(4)$ & $<0.0001$ \\
\hline Chronic pulmonary disease & $12.0(118)$ & $6.2(3)$ & $25.6(31)$ & $10.3(84)$ & $<0.0001$ \\
\hline Asthma & $5.2(51)$ & $0(0)$ & $6.6(8)$ & $5.3(43)$ & 0.2 \\
\hline COPD & $4.0(39)$ & $2.1(1)$ & $9.1(11)$ & $3.3(27)$ & 0.008 \\
\hline Chronic liver diseases & $3.4(33)$ & $6.2(3)$ & $13.2(16)$ & $1.7(14)$ & $<0.0001$ \\
\hline Thyroid diseases & $18.6(182)$ & $29.2(14)$ & $23.1(28)$ & $17.2(140)$ & 0.045 \\
\hline Venous thromboembolism & $3.5(34)$ & $8.3(4)$ & $7.4(9)$ & $2.6(21)$ & 0.004 \\
\hline Cancer & $10.3(101)$ & $2.1(1)$ & $35.5(43)$ & $7.0(57)$ & $<0.0001$ \\
\hline Dementia syndrome & $3.4(33)$ & $14.6(7)$ & $6.6(8)$ & $2.2(18)$ & $<0.0001$ \\
\hline \multicolumn{6}{|l|}{ Drugs, \% $(n)$ : } \\
\hline Aspirin & $26.4(259)$ & $58.3(28)$ & $44.6(54)$ & $21.8(177)$ & $<0.0001$ \\
\hline Anticoagulants (DOACs/VKA) & $9.8(96)$ & $22.9(11)$ & $21.5(26)$ & $7.3(59)$ & $<0.0001$ \\
\hline Clopidogrel & $6.9(68)$ & $25.0(12)$ & $12.4(15)$ & $5.0(41)$ & $<0.0001$ \\
\hline \multicolumn{6}{|l|}{ Procedures*, \% (n): } \\
\hline Coronary angiography & $10.0(98)$ & $27.1(13)$ & $19.0(23)$ & $7.6(62)$ & $<0.0001$ \\
\hline PTCA & $6.1(60)$ & $14.6(7)$ & $11.6(14)$ & $4.8(39)$ & 0.0006 \\
\hline
\end{tabular}

${ }^{a} A$ significantly greater value in the UA group than the NA group for the following parameters: hypertension and thyroid diseases. ${ }^{b} A$ significantly greater value in the UA group than the DA group and the UA group than the NA group for the following parameter: coronary heart disease. ${ }^{c}$ A significantly lower value in the NA group than the UA group and the DA group for the following parameters: chronic kidney disease, heart failure, atrial fibrillation, aspirin, anticoagulants (DOACS/VKA), coronary angiography, PTCA, chronic liver diseases, venous thromboembolism, dementia syndrome, clopidogrel. ${ }^{d} A$ significantly greater value in the DA group than the UA group for COPD. COPD - chronic obstructive pulmonary disease, DA - defined anemia, DOAC - direct oral anticoagulants, NA - no anemia, PTCA - percutaneous transluminal coronary angioplasty, UA - unexplained anemia, VKA - vitamin K antagonist. A p-value less than 0.05 was considered statistically significant. ${ }^{\star} T$ The subject of analysis was whether the procedure was performed in a patient at least once and not the number of procedures performed in the patient. 
When comparing the NA with the DA group, a large effect size was obtained in the case of hemoglobin, platelet count, CRP, the number of hospitalizations and the number of comorbidities ( $d$ : 0.84-2.87), while a medium effect size for GFR and the Barthel scale scores was obtained ( $d$ : 0.37-0.41). The difference between the UA group and the group without anemia demonstrated a large effect size for hemoglobin concentration and the number of comorbidities (d: 0.96-2.55) and a medium effect size for GFR, the number of hospitalizations and the Barthel scale scores ( $d$ : 0.39-0.71). In the case of CRP a medium effect size was found between the UA group and DA group $(d=0.24)$.

In patients with UA, the incidence of cardiovascular diseases (hypertension, ischemic heart disease, heart failure, atrial fibrillation, venous thromboembolism), CKD, chronic liver diseases, dementia syndrome and thyroid gland diseases was significantly higher than in the patients without anemia (Table II). Aspirin, clopidogrel and anticoagulants (DOACs/VKA) were also more frequently administered in the UA group. Coronary angiography and percutaneous transluminal coronary angioplasty (PTCA) were performed more often in patients with UA. There were a few differences between the UA and DA groups; these were significantly more frequent ischemic heart disease and the use of clopidogrel in the UA group.

\section{All-cause mortality}

Of the 981 patients, 40 (4.0\%) died during the 3-year follow-up, including 16 (13.2\%) patients with DA, 5 (10\%) with UA and 19 (2.3\%) in the group without anemia. In a Kaplan-Meier analysis, patients with UA and patients with DA had significantly lower overall survival rates as compared to patients without anemia $\left(\chi^{2}=29.54, p<0.001\right)$ (Figure 2). The survival of people in the UA vs. DA vs. NA group was $88 \%$ vs. $88 \%$ vs. $97 \%$ after

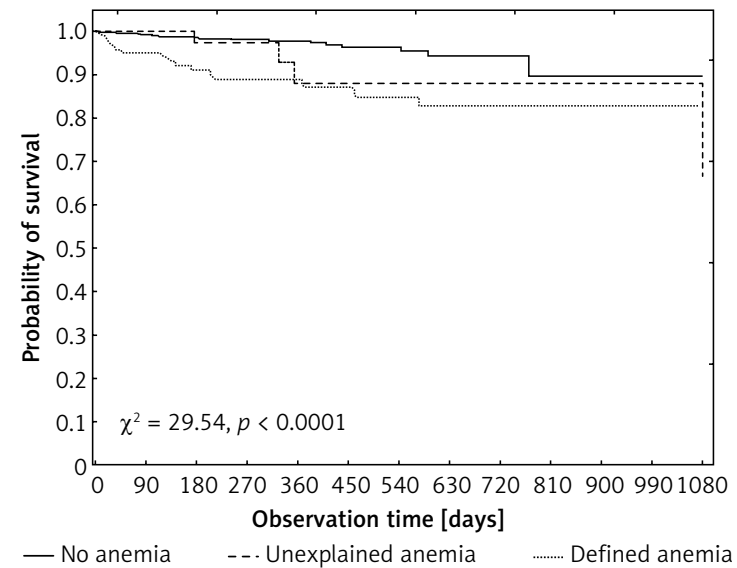

Figure 2. Kaplan-Meier analysis of overall survival rates in the analyzed groups
360 days, $87 \%$ vs. $83 \%$ vs. $94 \%$ after 720 days and $87 \%$ vs. $83 \%$ vs. $89 \%$ at the time of follow-up (after 1080 days), respectively. No significant differences were observed in survival rates in the 3-year follow-up, between patients with UA and DA.

\section{Discussion}

In this retrospective analysis of 981 patients aged $\geq 60$ years, unexplained anemia was found in $4.9 \%$ of the total studied population and in $28.4 \%$ of patients diagnosed with anemia. These results are compatible with other studies [5-10]. In our study, the prevalence of UA increased with age, being the highest in the population $\geq 80$ years. To our knowledge, the prevalence of UA depending on age has not been previously analyzed. However, an increase in the incidence of anemia with age is well documented $[1,9,18,19]$, as it is associated with the process of aging and deterioration in the functioning of a number of organs, including bone marrow, kidneys, the immune system and deficiencies of sex hormones [10, 20, 21]. Bone marrow disorders, observed in the elderly, are considered to be particularly important, as myelodysplastic syndrome (MDS) occurs in 5-15\% of those with anemia or other cytopenias [5, 7, 9, 19]. Therefore, the presence of cytopenias, after excluding vitamin $B_{12}$ or folic acid deficiencies, should lead to the diagnosis of MDS or other clonal diseases. In our study $81 \%$ of the patients with unexplained anemia lacked adequate hematological diagnostics. Bone marrow examination and cytogenetic/molecular tests were not performed in any of these patients, probably due to the mild grade of the anemia and the invasiveness of the procedure. It also seems that the presence of anemia in elderly patients is overlooked by doctors. Since almost $19 \%$ of the UA patients had leukopenia and/or thrombocytopenia or macrocytosis, MDS was the possible reason for anemia, at least in some cases. It was interesting that incomplete diagnosis of anemia was significantly more common in the youngest group of patients (6069 years). A possible explanation is the number of comorbidities increasing with age, which means more frequent medical consultations and hospitalizations, increasing the likelihood of diagnosis of anemia. Patients with UA had more comorbidities and more often suffered from cardiovascular disease (hypertension, coronary heart disease, heart failure and atrial fibrillation), chronic kidney disease, thyroid diseases, chronic liver diseases, venous thromboembolism and dementia syndrome. The UA degree in the elderly was found to be mild to moderate, as in other studies [10, 20, 21 ], and diagnosis of anemia was often incomplete in both out- and in-patients [22-24]. In the study of Artz et al., only $31 \%$ of patients with UA 
and other associated hematological abnormalities underwent bone marrow examination, and consequently myeloid neoplasms were diagnosed in $7.5 \%$ of patients with anemia, including MDS or acute myeloid leukemia in 5\% of patients [7]. Molecular studies confirmed that somatic mutations were observed more often in patients with cytopenia in comparison to the healthy population; however, the same observation concerned people older than 75 [12]. It is also well known that there is a higher risk of hematological malignancies if multiple mutations, especially at high frequency, are present in patients with cytopenia $[12,25]$. Despite some uncertainty, clinical testing for mutation is reasonable in the case of UA, especially in this population $[11,12]$. One should be aware however that in routine practice, cytogenetic/molecular and other tests in all elderly patients usually frail, with mild UA probably not being possible mainly due to the invasiveness and high costs. Bone marrow biopsy in elderly patients with mild anemia is controversial and discussed in the literature. According to Abrahamsen et al., invasive diagnostics are not necessary in patients with mild UA, and attentive observation is sufficient [13]. For now, it seems that research in patients with UA on the role of disorders related to notch signaling or long chain non-coding RNA is the future in prevention, prognosis or treatment, as is the case of many age-related diseases, including hematological malignancies and other cancers [26-28]. In our study however, during a 3-year follow-up, significantly more deaths were noted in the group with UA than in the group without anemia, even though the anemia was mild in most of the cases. The impact of UA on survival rates in elderly patients has only been analyzed in a few studies, with divergent results. The reasons for these discrepancies might be differences in the study populations in terms of age (from $>50$ to $\geq 85$ years) and other clinical characteristics, such as concomitant diseases, the median concentration of hemoglobin and the duration of follow-up (from 1 to 10 years) $[8,13,14,29]$. The impact of UA on mortality in different populations of elderly patients would be a key issue in the context of the need for detailed diagnostic procedures. This requires further studies taking into account different age ranges and longer follow-ups.

In our study, patients with UA, as compared to those without anemia, were more burdened with comorbidities, more frequently hospitalized and more frequently used ASA, anticoagulants and clopidogrel. There were also differences between patients with UA and DA, including more frequent ischemic heart disease and more frequent use of clopidogrel in the group with UA. It has been shown in many studies that patients with even mild anemia have more comorbidities, especially cardiovas- cular and kidney diseases and diabetes $[1,5,13$, 30-32], and more often require hospitalization [1, $2,30,33]$, as compared to patients without anemia. Patients with UA, due to the lack of a known cause, often for many years have lower hemoglobin values, which may contribute to the progression of cardiovascular disease. Perhaps for this reason, patients with UA in our study suffered from ischemic heart disease more often than patients with defined anemia (which may be treated).

Patients with UA suffered more often from a dementia syndrome and attained a lower score on the Barthel scale than patients without anemia, as was also previously reported in other studies $[13,34]$. The Barthel scale was implemented more frequently in individuals with anemia, which indicates their worse condition, as the Barthel scale is used in Poland to establish indications for long-term care (both stationary and home). It can be assumed that inferior physical and cognitive status might contribute to inadequate diagnosis of anemia, especially in the case of UA, where specialist consultations, invasive procedures, or even hospitalization are required.

In almost $17 \%$ of patients with UA in the present analysis, surgery was performed within 3 months before the diagnosis of anemia. In the literature, hospital-acquired anemia (HAA) is mentioned as one of the main causes of anemia [35], and it is probably one of the most ignored causes of anemia in the elderly.

In this analysis, we used the definition of anemia according to WHO criteria from 1968. A wide discussion on the hemoglobin threshold exists in the literature [2, 3, 14, 36, 37], it being suggested by some authors that the normal $\mathrm{Hb}$ value in elderly patients is too high [14, 37]. On the other hand, many studies indicate that anemia, even at a mild degree, increases mortality and morbidity in the elderly $[1-3,38]$.

Our research has limitations resulting from its retrospective character and incomplete data including erythrocyte indices. Indeed, a large group of patients (981) were included in the study, but patients with UA constituted only 48 patients. In addition, patients with UA in relation to those without anemia were older and had more comorbidities, which could have affected their inferior survival rates. While anemia is a symptom of many diseases, it can also influence the course of many diseases, including atherosclerosis and cardiovascular diseases [39, 40], and thus increase the risk of death. It seems that anemia - even mild in an elderly patient - is an alarm symptom, indicating increased mortality.

In conclusion, UA is a common problem in the population aged $\geq 60$ years. It accounted for almost one third of the anemia cases in this retrospective analysis and its prevalence increased 
with age. Despite the fact that the anemia was mild in the majority of patients with $U A$, the results of our study demonstrated higher mortality, worse performance status assessed with the Barthel scale, and more comorbidities and hospitalizations in the group with UA as compared to the group without anemia. We have shown that UA was insufficiently diagnosed in the elderly Polish population. Establishing a diagnosis would allow for causal treatment of anemia, whenever possible, resulting in an improvement of patients' outcome. There is a need for further research on anemia in the elderly not only to identify the causes, but also to develop recommendations for diagnostic procedures and treatment of undefined anemia, for hematologists as well as for primary care physicians.

\section{Conflict of interest}

The authors declare no conflict of interest.

\section{References}

1. Penninx BWJH, Pahor M, Woodman RC, Guralnik JM. Anemia in old age is associated with increased mortality and hospitalization. J Gerontol A Biol Sci Med Sci 2006; 61: 474-9.

2. Culleton BF, Manns BJ, Zhang J, Tonelli M, Klarenbach S, Hemmelgarn BR. Impact of anemia on hospitalization and mortality in older adults. Blood 2006; 107: 3841-6.

3. Zakai NA, Katz R, Hirsch C, et al. A prospective study of anemia status, hemoglobin concentration, and mortality in an elderly cohort: the Cardiovascular Health Study. Arch Intern Med 2005; 165: 2214-20.

4. Beghé C, Wilson A, Ershler WB. Prevalence and outcomes of anemia in geriatrics: a systematic review of the literature. Am J Med 2004; 116 Suppl 7A: 3S-10S.

5. Guralnik JM, Eisenstaedt RS, Ferrucci L, Klein HG, Woodman RC. Prevalence of anemia in persons 65 years and older in the United States: evidence for a high rate of unexplained anemia. Blood 2004; 104: 2263-8.

6. Anía BJ, Suman VJ, Fairbanks VF, Rademacher DM, Melton LJ. Incidence of anemia in older people: an epidemiologic study in a well defined population. J Am Geriatr Soc 1997; 45: 825-31.

7. Artz AS, Thirman MJ. Unexplained anemia predominates despite an intensive evaluation in a racially diverse cohort of older adults from a referral anemia clinic. J Gerontol A Biol Sci Med Sci 2011; 66: 925-32.

8. Shavelle RM, MacKenzie R, Paculdo DR. Anemia and mortality in older persons: does the type of anemia affect survival? Int J Hematol 2012; 95: 248-56.

9. Joosten E, Pelemans W, Hiele M, Noyen J, Verhaeghe R, Boogaerts MA. Prevalence and causes of anaemia in a geriatric hospitalized population. Gerontology 1992; 38: 111-7.

10. Ferrucci L, Guralnik JM, Bandinelli S, et al. Unexplained anaemia in older persons is characterised by low erythropoietin and low levels of pro-inflammatory markers. Br J Haematol 2007; 136: 849-55.

11. Stauder R, Valent P, Theurl I. Anemia at older age: etiologies, clinical implications, and management. Blood 2018; 131: 505-14.
12. Steensma DP. New challenges in evaluating anemia in older persons in the era of molecular testing. Hematol Am Soc Hematol Educ Program 2016; 2016: 67-73.

13. Abrahamsen JF, Monsen ALB, Landi F, Haugland C, Nilsen RM, Ranhoff AH. Readmission and mortality one year after acute hospitalization in older patients with explained and unexplained anemia - a prospective observational cohort study. BMC Geriatr 2016; 16: 109.

14. Willems JM, den Elzen WPJ, Vlasveld LT, et al. No increased mortality risk in older persons with unexplained anaemia. Age Ageing 2012; 41: 501-6.

15. Michalak SS, Rupa-Matysek J, Gil L. Comorbidities, repeated hospitalizations, and age $\geq 80$ years as indicators of anemia development in the older population. Ann Hematol 2018; 97: 1337-47.

16. Blanc B, Finch C, Hallberg L, Herbert V. Nutritional anaemias. Report of a WHO scientific group. World Health Organ Tech Rep Ser 1968; 405: 5-37.

17. Mahoney Fl, Barthel DW. Functional evaluation: the Barthel Index. Md State Med J 1965; 14: 61-5.

18. Gaskell H, Derry S, Andrew Moore R, McQuay HJ. Prevalence of anaemia in older persons: systematic review. BMC Geriatr 2008; 8: 1.

19. Bach V, Schruckmayer G, Sam I, Kemmler G, Stauder R. Prevalence and possible causes of anemia in the elderly: a cross-sectional analysis of a large European university hospital cohort. Clin Interv Aging 2014; 9: 1187-96.

20. Waalen J, von Löhneysen K, Lee P, Xu X, Friedman JS. Erythropoietin, GDF15, IL6, hepcidin and testosterone levels in a large cohort of elderly individuals with anaemia of known and unknown cause. Eur J Haematol 2011; 87: 107-16.

21. Artz AS, Xue QL, Wickrema A, et al. Unexplained anaemia in the elderly is characterised by features of low grade inflammation. Br J Haematol 2014; 167: 286-9.

22. Beloosesky Y, Cohen AM, Grosman B, Grinblat J. Prevalence and survival of myelodysplastic syndrome of the refractory anemia type in hospitalized cognitively different geriatric patients. Gerontology 2000; 46: 323-7.

23. Silverberg DS, Schwartz D, Schwartz I, Ben Assa E. The missed opportunities to diagnose and treat iron deficiency in patients hospitalized with heart failure. Int J Cardiol 2013; 168: 2164-6.

24. Tang WHW, Tong W, Jain A, Francis GS, Harris CM, Young JB. Evaluation and long-term prognosis of new-onset, transient, and persistent anemia in ambulatory patients with chronic heart failure. J Am Coll Cardiol 2008; 51: 569-76.

25. Malcovati L, Gallì A, Travaglino E, et al. Clinical significance of somatic mutation in unexplained blood cytopenia. Blood 2017; 129: 3371-8.

26. Balistreri CR, Madonna R, Melino G, Caruso C. The emerging role of Notch pathway in ageing: focus on the related mechanisms in age-related diseases. Ageing Res Rev 2016; 29: 50-65.

27. Jędroszka D, Orzechowska M, Bednarek AK. Predictive values of Notch signalling in renal carcinoma. Arch Med Sci 2017; 13: 1249-54.

28. Kang K, Huang YH, Li HP, Guo SM. Expression of UCA1 and MALAT1 long-chain non-coding RNAs in esophageal squamous cell carcinoma tissues is predictive of patient prognosis. Arch Med Sci 2018; 14: 752-9.

29. Semba RD, Ricks MO, Ferrucci L, et al. Types of anemia and mortality among older disabled women living in the community: the Women's Health and Aging Study I. Aging Clin Exp Res 2007; 19: 259-64.

30. Young JB, Abraham WT, Albert NM, et al. Relation of low hemoglobin and anemia to morbidity and mortality in 
patients hospitalized with heart failure (insight from the OPTIMIZE-HF registry). Am J Cardiol 2008; 101: 223-30.

31. Lawler PR, Filion KB, Dourian T, Atallah R, Garfinkle M, Eisenberg MJ. Anemia and mortality in acute coronary syndromes: a systematic review and meta-analysis. Am Heart J 2013; 165: 143-53.e5.

32. Gandhi SJ, Hagans I, Nathan K, Hunter K, Roy S. Prevalence, comorbidity and investigation of anemia in the primary care office. J Clin Med Res 2017; 9: 970-80.

33. Lucca U, Tettamanti M, Mosconi P, et al. Association of mild anemia with cognitive, functional, mood and quality of life outcomes in the elderly: the "Health and Anemia" study. PloS One 2008; 3: e1920.

34. Hong CH, Falvey C, Harris TB, et al. Anemia and risk of dementia in older adults. Neurology 2013; 81: 528-33.

35. Kurniali PC, Curry S, Brennan KW, et al. A retrospective study investigating the incidence and predisposing factors of hospital-acquired anemia. Anemia 2014; 2014 634582.

36. Beutler E, Waalen J. The definition of anemia: what is the lower limit of normal of the blood hemoglobin concentration? Blood 2006; 107: 1747-50.

37. Andrès E, Serraj K, Federici L, Vogel T, Kaltenbach G. Anemia in elderly patients: new insight into an old disorder Geriatr Gerontol Int 2013; 13: 519-27.

38. Riva E, Tettamanti M, Mosconi P, et al. Association of mild anemia with hospitalization and mortality in the elderly: the health and anemia population-based study. Haematologica 2009; 94: 22-8.

39. Rymer JA, Rao SV. Anemia and coronary artery disease: pathophysiology, prognosis, and treatment. Coron Artery Dis 2018; 29: 161-7.

40. Michalska-Kasiczak M, Bielecka-Dabrowa A, von Haehling S, Anker SD, Rysz J, Banach M. Biomarkers, myocardial fibrosis and co-morbidities in heart failure with preserved ejection fraction: an overview. Arch Med Sci 2018; 14: 890-909. 